\title{
INTERNET, HAUT LIEU \\ DE LA RADICALISATION
}

inguistiquement, le terme «radicalisation» est issu du latin radix

qui signifie « racine, origine». L'adjectif «radical» provient du latin tardif radicalis qui qualifie toute action visant un retour aux sources, en faisant table rase de l'existant. Ainsi, la radicalisation désigne un processus à la fois intellectuel et actionnel refusant le compromis et la conciliation, associé à une volonté de purification des individus et des sociétés, expurgeant tout ce qui est perçu comme «mauvais» ou «corrupteur».

De ce point de vue, il existe une distinction de fond entre la radicalisation en tant que «processus» et le terrorisme en tant que «produit». La radicalisation se situe en amont, le terrorisme en aval, c'est-à-dire que l'une peut mener à l'autre mais pas nécessairement. Cette distinction est essentielle d'un point de vue conceptuel car les protagonistes ne sont pas les mêmes. Dans un cas, il s'agit d'acteurs (de la radicalisation), dans l'autre de vecteurs (de violence). Cette nuance permet de suivre l'évolution de la radicalisation et sa traduction en extrémisme violent ou en actions terroristes sur le terrain.

En effet, s'agissant du terrorisme, les vecteurs de violence sont des groupes armés et des organisations bien identifiées qui font quotidiennement les titres de l'actualité tels que: l'organisation État islamique (EI), Al-Qaïda, Boko Haram, les shebab somaliens, etc. En revanche, pour ce qui est de la radicalisation, les acteurs sont plus flous et moins bien connus. Car il s'agit tout à la fois de courants religieux, de doctrines théologiques, d'associations cultuelles, ou encore de leaders politicoreligieux qui participent, délibérément ou inconsciemment, au processus de radicalisation dans les sociétés ciblées par leur action de propagande.

Certes, analysée à travers le prisme exclusivement sécuritaire, la radicalisation ne peut être dissociée du terrorisme, mais cela ne doit pas faire 
oublier la distinction de fond entre le processus et le produit, ni entre les acteurs et les vecteurs. Car cette confusion peut s'avérer, par la suite, préjudiciable à l'analyse intellectuelle de la radicalisation comme à l'action politique de lutte contre le terrorisme.

Ce cadrage conceptuel exige également d'insister sur la différence existant entre la définition théorique de la radicalisation et sa perception dans la population. En effet, il existe de nombreux cas où il peut s'agir, par exemple, de simple expression identitaire (port du voile), mais une telle expression peut être perçue comme la manifestation d'une radicalisation religieuse. Les exemples de «radicalisation perceptive» ne manquent pas et suscitent, parfois, des réactions de rejet, de stigmatisation ou encore de discrimination, lesquelles nourrissent par ricochet toutes sortes d'extrémismes.

L'analyse de la radicalisation doit donc tenir compte de la perception collective, c'est-à-dire de l'état de l'opinion publique à un moment donné et de son degré de tolérance à l'égard de certaines expressions ou manifestations de la radicalité. L'on constate, par exemple, que le recours à la violence dans les actions syndicalistes est de moins en moins «toléré» au sein de la société française, même s'il continue d'être largement répandu chez certaines catégories socioprofessionnelles.

Il existe ainsi une porosité des frontières entre la radicalisation idéologique et le radicalisme politique. Au sein même des démocraties occidentales, certains hommes politiques d'extrême droite (Donald Trump par exemple) font des déclarations apparentées à celles des leaders extrémistes. Par ailleurs, dans les pays où l'on assiste à une résurgence du religieux, il est difficile de faire le tri entre les courants qui relèvent de l'intégrisme et du fondamentalisme, et ceux qui relèvent de la dérive sectaire ou encore de l'extrémisme violent.

Mais l'expérience de terrain montre que la radicalisation n'est pas générale et qu'elle concerne seulement certains «territoires» et certains «foyers». En revanche, il parait difficile de «localiser» cette radicalisation de façon précise, parce qu'elle emprunte pour sa propagation la voie d'internet et des réseaux sociaux. C'est pourquoi l'on parle de plus en plus de « radicalisation virtuelle».

\section{L'ESSOR DE LA RADICALISATION SUR INTERNET}

$\mathrm{La}$ «radicalisation virtuelle» désigne le processus de basculement dans la violence qui intervient via le média internet. Ce dernier a en effet beaucoup évolué et profondément modifié les comportements et les 
rapports de force au cours des dernières années. Après les blogs et les forums des années 2000, c'est le règne des réseaux sociaux et des communautés virtuelles. Celles-ci désignent un groupe de personnes qui communiquent par l'intermédiaire d'internet en fonction de centres d'intérêt et d'affinités particulières. La virtualité signifie seulement que la communication ne se fait pas en face à face mais par le biais d'un ordinateur ou d'un smartphone.

Cette évolution techno-sociale a donné lieu à une «internetisation » du terrorisme, autrement dit à un processus de transformation d'internet en canal privilégié de propagande et de recrutement en faveur des organisations terroristes, au premier rang desquelles figure l'EI, plus connu en français sous l'acronyme Daech.

Ces communautés virtuelles constituent une évolution majeure d'internet car elles offrent un lieu d'échange et de partage entre individus reliés par des interactions verbales et/ou audiovisuelles qui peuvent faire basculer dans la radicalisation ou mener au terrorisme. Il existe ainsi d'innombrables applications sur internet qui permettent de se trouver des «amis », de suivre un «leader», de créer son propre «réseau social» dédié à la promotion de l'action violente ou même des organisations terroristes.

Cette virtualisation de la violence concerne l'ensemble des technologies et des usages du World Wide Web, en particulier des fonctionnalités inédites permettant aux internautes d'interagir de façon rapide et anonyme, via des pseudonymes et des réseaux sécurisés, créant ainsi des formes nouvelles de communication «participative» qui nourrissent un «web subversif» de plus en plus important. Cette dernière appellation fait référence au média internet considéré comme un espace de contestation radicale, un lieu dont l'une des fonctions principales est la promotion d'une «culture révolutionnaire» tournée vers l'action violente, et non plus uniquement vers la mise à disposition d'informations ou de documents pour la simple consultation des internautes.

Ainsi, l'évolution du terrorisme au cours des dernières années suit de près les progrès de la technologie et en particulier d'internet, de sorte qu'il n'est pas exagéré de parler aujourd'hui d'une génération de terroristes apparue dans la foulée du «web 2.0» caractérisée, comme lui, par ses réseaux sociaux et par ses leaders virtuels. Et comme l'on évoque déjà l'avènement du «web 3.0", voire du «web 4.0 », il y aura probablement une nouvelle génération de terroristes pour accompagner chaque version nouvelle du monde virtuel construit par et autour d'internet. 


\section{LE PROCESSUS DE RADICALISATION SUR INTERNET}

À l'origine de la radicalisation se trouve toujours un processus complexe de politisation des faits et des événements, processus par lequel un individu est amené à s'intéresser à la (géo)politique et à développer des réflexions spécifiques concernant un conflit ou une controverse. Progressivement, les questions politiques pénètrent le quotidien de l'individu et accaparent son intérêt et son attention, le poussant vers une politisation de plus en plus affirmée.

Dans cette politisation, tout est perçu et analysé en termes de conflictualité et de rapports de force au sein desquels une puissance hégémonique exerce sa domination sur un groupe ou une communauté dominés. Les événements du monde ne sont pas reçus dans l'indifférence ni même à travers le canal médiatique habituel, mais réinterprétés suivant une grille personnelle faite de tiraillement entre le constat de la domination et l'aspiration à la révolte.

Ainsi par exemple, sur les réseaux djihadistes, les événements en Syrie ne sont pas reçus aujourd'hui comme le déroulement sordide d'une guerre civile qui fait rage depuis des années, mais interprétés comme un pur jeu des puissances occidentales et régionales visant à détruire la Syrie et à exterminer les musulmans de façon délibérée. De même, la situation chaotique en Irak n'est pas imputée aux acteurs locaux mais à l'ingérence étrangère. Entre les théories du complot et la paranoïa ambiante, la réalité virtuelle ne fait qu'aggraver les tendances conspirationnistes de certains individus radicalisés.

Au niveau individuel justement, cette radicalisation perceptive se traduit par des formes de militantisme manifeste chez certains citoyens, convaincus de contribuer à une «guerre juste». L'on a vu ainsi se multiplier les expressions d'adhésion à la «cause syrienne», avant que l'engagement militant ne se «confessionnalise» à son tour, conduisant à l'engagement de ces mêmes individus aux côtés des groupes islamistes, devenus entretemps la principale force d'opposition armée au régime syrien.

Le passage du militantisme à l'activisme s'est d'abord manifesté sur internet par des formes multiples de propagande en faveur de l'un ou l'autre camp. À travers cette propagande, les réseaux sociaux ont joué un rôle central d'ancrage du conflit dans les consciences et de renforcement du clivage confessionnel dans les discours. D’un côté, les activistes pro-chiites ont tout mis en ouvre pour redorer le blason du régime syrien et le présenter comme «seul rempart contre le terrorisme », voire comme «défenseur de la laïcité». D’un autre côté, les activistes pro-sunnites se 
sont évertués à montrer que les groupes de l'opposition armée n'avaient d'islamiste que le nom et que leur objectif final était l'instauration d'un régime plus juste. Bien évidemment, des deux côtés, la présentation des faits était biaisée et le résultat loin de la réalité, où des centaines de civils innocents tombaient tous les jours sous les coups des deux camps.

Dans les pays occidentaux, cet activisme a eu pour effet d'importer le conflit et de cliver les militants entre partisans et opposants à l'intervention militaire, puis rapidement entre pro- et anti-islamistes. Le fait que l'opposition syrienne läque ait refusé d'enrôler les volontaires occidentaux dans ses rangs pour préserver le soutien diplomatique de leurs gouvernements a jeté ces combattants dans les bras des organisations les plus radicales, avant d'en faire de véritables djihadistes.

Sur les réseaux sociaux, l'activisme politique s'est accompagné d'une radicalisation théologique faisant la promotion de croyances mortifères axées sur le «djihad» et le «martyre». Progressivement, bon nombre de militants occidentaux qui voulaient «faire quelque chose» pour aider le peuple syrien se sont retrouvés pris dans la logique implacablement confessionnelle de la guerre civile. Mus originellement par des raisons «humanitaires», ils ont dérivé vers des actions violentes menées au nom de la religion.

Cette radicalisation actionnelle signe le passage de l'activisme au terrorisme, induisant un ciblage des populations civiles en fonction de critères confessionnels. Ainsi, les chiites sont ciblés parce qu'ils sont chiites et les sunnites sont tués parce qu'ils sont sunnites. Les milices des deux camps sévissent partout dans le pays, accentuant le clivage confessionnel et suscitant davantage de compassion et d'adhésion au sein de leur communauté d'origine. C'est la logique infernale de la discrimination et de la communautarisation, qui trouve son expression la plus virulente sur les réseaux sociaux et internet.

\section{LE TERRORISME ET INTERNET}

Aujourd'hui, les outils du web sont à la portée de tous les insurgés et de tous les apprentis terroristes de la planète, lesquels n'hésitent pas à en faire une exploitation maximale, à l'instar de n'importe quel utilisateur averti d'internet, du Maroc au Pakistan, en passant par la Syrie et le Yémen. Aussi, la question de fond posée concernant le terrorisme actuel n'est pas tant celle de la propagande diffusée sur internet que, plus précisément, celle de son appropriation par les internautes non affiliés à la mouvance djihadiste. Cet intérêt se manifeste à travers les téléchargements 
les plus fréquents, les commentaires postés sur les réseaux sociaux au sujet d'un attentat ou d'une séquence vidéo, ou encore les expressions de sympathie ou d'adhésion au sujet de tel ou tel document (écrit, audio ou vidéo) posté par des organisations terroristes.

En effet, la démocratisation de l'accès à l'information sur internet et l'accessibilité des réseaux de partage dans la plupart des langues internationales fait que tout un chacun peut lire les textes de propagande ou visionner des vidéos postées par des djihadistes ou par des sympathisants d'organisations terroristes partout où il se trouve. Le problème est, par conséquent, celui de l'impact de ce contenu audiovisuel sur les individus qui le consultent régulièrement. On sait, à partir de leurs réactions spontanées, qu'il s'agit là d'un élément clé du processus de radicalisation. Cela joue le rôle d'un facilitateur d'auto-endoctrinement et d'auto-embrigadement. L'exemple type est celui des adolescent(e)s choqué(e)s, voire traumatisé(e)s par des vidéos violentes mettant en scène des enfants. Progressivement, les images des massacres les obsèdent, les empêchant parfois de dormir, et les entraînent dans un cycle infernal où le désir de s'affirmer les pousse dans les bras d'organisations terroristes qui parviennent ainsi à attirer les jeunes les plus désorientés.

Il existe, à cet égard, un grand nombre d'exemples d'individus qui ont reconnu avoir utilisé internet de façon intensive dans leur processus de radicalisation avant de basculer dans le terrorisme ou bien de rejoindre des organisations extrémistes. C'est le cas de la majorité des jeunes Européens qui ont rejoint l'EI en Syrie et en Irak. Tous avouent le grand impact des réseaux sociaux sur leur psychologie et son rôle central dans leur endoctrinement.

\section{L'ORGANISATION ÉTAT ISLAMIQUE ET INTERNET}

Même si l'EI attire des informaticiens et des recrues ayant des compétences technologiques, il semble encore incapable de mettre en œuvre des attaques cyberterroristes d'envergure. En avril 2015, la cyberattaque qui avait visé Tv5 Monde, initialement attribuée à l'EI, s'est avérée être l'œuvre des hackers russes réagissant, en pleine guerre d'Ukraine, au traitement de la crise par la chaîne internationale d'expression française.

En revanche, l'EI continue d'innover en matière de propagande sur internet et utilise l'ensemble des technologies et des outils disponibles pour augmenter sa présence et son effet sur le web. L'organisation ne met pas seulement, à disposition des internautes, des informations ou des documents via les réseaux sociaux, elle y recourt également pour 
construire des «équipes de choc» chargées de relayer son message et de promouvoir son action dans le monde entier ${ }^{1}$.

Le fait que la communication ne se fait pas en face à face ne signifie pas que les communautés sont purement fictives ou virtuelles; bien au contraire, elles ont souvent des prolongements dans la réalité et abritent des activités dont certaines sont illégales ou génératrices de radicalisation ou de délinquance. En effet, la génération actuelle est nourrie au sein technologique et médiatique, mais elle n'a pas nécessairement l'appareillage critique lui permettant d'appréhender la complexité et le foisonnement des informations qui s'offrent à elle dans le monde virtuel concernant des crises et des conflits bien réels.

Le fait que l'EI communique dans la plupart des langues internationales explique notamment la multiplication des déclarations d'allégeance à distance, avec la montée en puissance de l'EI et sa déclaration du «Califat» à partir de l'été 2014. Dès lors, des figures du web sont apparues pour relayer le message de l'organisation et recruter de nouveaux partisans. Ces propagandistes sont repérables à la fréquence et à la nature de leurs contributions. Dans la plupart des cas, il s'agit d'individus issus des pays européens qui recrutent à distance parmi leurs compatriotes.

Les ressorts psychologiques sur lesquels jouent ces propagandistes sont ceux du ressentiment à l'égard de l'Occident, accusé de tous les maux des musulmans. Mais, du côté de l'internaute et notamment de certains jeunes en pleine crise d'adolescence, c'est surtout le désir intégriste de pureté et de justice qui motive, la plupart du temps, le basculement dans la radicalisation. En voulant défendre les «frères opprimés» ou encore «venger les victimes musulmanes », l'internaute opère une déshumanisation de ses concitoyens qui légitime, par ricochet dans son esprit tourmenté, le fait de les prendre comme cibles. En réalité, c'est une dynamique psychologique paradoxale qui conduit celui qui veut «la justice» à devenir l'instrument le plus brutal de «l'injustice».

Certains de ces recruteurs ne se contentent pas de messages ponctuels mais jouent un rôle prédominant dans l'animation des réseaux et des communautés virtuelles. Ils répondent aux questions des internautes en fournissant des informations pratiques, des éclaircissements théologiques, des documents particuliers, ou encore des astuces techniques. Lorsqu'ils ne répondent pas directement à la requête d'un internaute, ils jouent un rôle d'orientation grâce aux conseils qu'ils prodiguent sur les questions posées: recommandation de lectures, de visites de sites

1. Cf. Mathieu Guidère, L'État islamique en 100 questions, Paris, Tallandier, 2016. 
web, de connexion avec d'autres membres, etc. Ainsi, par exemple, les demandes d'aide à «l'émigration» (bijra) vers le «territoire du Califat» reçoivent systématiquement une réponse brève de renvoi vers une autre page du réseau sur laquelle l'internaute peut trouver tous les éléments de réponse à sa question.

Enfin, ces recruteurs agissent comme des animateurs de réseau, reconnaissables au fait que la plupart de leurs messages postés reprennent des informations (sous forme de retweets par exemple) ou de réactions à des articles publiés sur l'organisation. Ces modes d'interaction permettent de s'assurer le contrôle discursif du réseau ou de la communauté, en veillant à ce que les thématiques restent centrées sur les actions des «soldats du Califat». Aucun autre sujet ne doit détourner les membres de cette préoccupation.

Ainsi, l'étude des réseaux et des communautés virtuelles associées à l'Ei permet de mettre en évidence l'existence d'un système de recrutement élaboré. Ce système, même s'il s'exerce prioritairement dans le monde virtuel (sur internet), possède des prolongements dans le monde réel, lesquels se traduisent parfois par des actions terroristes revendiquées par l'EI.

Bref, la consommation individuelle de matériau djihadiste, librement disponible sur le web, que ce soit par une recherche personnelle ciblée ou bien au gré d'une navigation aléatoire dans l'océan de vidéos radicales, est le premier stade de la radicalisation violente. La quantité des données diffusées en ligne est aujourd'hui telle qu'apprendre à utiliser une arme ou à fabriquer une bombe sur internet est devenu un jeu d'enfant ou presque. La matière pédagogique ne manque pas pour quiconque voudrait se former à l'élaboration d'explosifs en tous genres.

Mais, si les «consommateurs» de ce matériau en ligne sont majoritairement issus des pays européens et anglo-saxons, les "producteurs", quant à eux, se trouvent généralement sur les «territoires du Califat». Le web, en raison d'un défaut de régulation internationale, continuera pendant longtemps à alimenter les rangs des organisations terroristes. 
Internet apparaît aujourd'bui comme le principal vecteur de radicalisation conduisant au terrorisme. L'outil en soi n'est pas en cause, il représente une évolution phare de notre temps; ce sont les usages subversifs qui posent problème. Aussi, internet est devenu l'instrument de propagande privilégié des organisations terroristes et le principal lieu de radicalisation des jeunes. Après une mise au point concernant le phénomène de la radicalisation virtuelle, cet article donne un aperçu des usages terroristes d'internet. 